\title{
High temperature permeability of fibrous materials using direct simulation Monte Carlo
}

\author{
Arnaud Borner ${ }^{\mathrm{a}, \mathrm{b}, *}$, Francesco Panerai ${ }^{\mathrm{c}, * *}$, Nagi N. Mansour ${ }^{\mathrm{b}}$ \\ ${ }^{a}$ Department of Mechanical Science and Engineering, University of Illinois \\ Urbana-Champaign, 4417 Mechanical Engineering Laboratory MC-144, 1206 West Green \\ Street, Urbana, IL, 61801, USA. \\ ${ }^{b}$ Computational Physics Branch, NASA Ames Research Center, Mail Stop 258-5, Moffett \\ Field, CA 94035, USA. \\ ${ }^{c}$ Department of Mechanical Engineering, University of Kentucky, 151 Ralph G. Anderson \\ Bldg., Lexington, KY, 40506, USA.
}

\begin{abstract}
Porous carbon fiber materials are used as effective insulators in many applications where high temperatures are involved. In particular, they are used as the substrate of ablative thermal protection materials for atmospheric entry systems. In this application and in many other industrial uses, quantifying the permeability of porous materials is needed to compute the flow rate of gases through them, under certain environmental conditions. In this work, direct simulation Monte Carlo (DSMC) simulations are used to compute permeability of several fibrous substrates to high temperature gases. The actual porous geometry of the materials is digitized using X-ray microtomography. Numerical results at various pressures and Knudsen numbers are compared with experimental data published in the literature. The method confirms that the pressure dependence of effective gas permeability is well represented by the Klinkenberg formulation. The method is validated by showing close agreement between measurements of permeability from simulations and experimental investigations. Four carbon fiber materials with different microstructures are investigated. We show that the permeability strongly depends on the pore size distribution, as well as on
\end{abstract}

\footnotetext{
* Corresponding author

** Current address: AMA Inc. at NASA Ames Research Center, Mail Stop 234-1, Moffett Field, CA 94035, USA.

Email address: arnaud.p.borner@nasa.gov (Arnaud Borner)
}

Preprint submitted to International Journal of Heat and Mass Transfer October 12, 2016

(C) 2016. This manuscript version is made available under the Elsevier user license http://www.elsevier.com/open-access/userlicense/1.0/ 
the porosity of the material.

Keywords: DSMC, Carbon fiber, Permeability, Porosity

\section{Introduction}

The hypersonic entry of a spacecraft in a planetary atmosphere requires Thermal Protection Systems (TPS) able to manage the heat received from freestream plasma. For very high entry speeds, ablators are the only option

5 as TPS materials. A successful class among the ablative materials family are lightweight porous materials based on a fibrous carbon insulator impregnated with phenolic. As these materials are highly porous, when performing material response simulations, one needs to account for the transport of gases through the microstructure. Gas transport through a porous material is regulated by the characteristic scale of the microstructure and that of the mean free path of the percolating flow. A parameter used to quantify this transport is the material permeability. Instances such as the flow of pyrolysis gas products, the penetration of hot boundary-layer gases, or the use of transpiration-cooling systems highlight the importance of internal gas transport in TPS materials. The gas permeability of a material must be known before the gas flow through it can be properly modeled. In general, data regarding the permeabilities of porous ablators is limited and challenging to measure experimentally.

In this paper we use a particle-based method, direct simulation Monte Carlo (DSMC) [1], to compute the permeability of low-density carbon fiber materials based on their digitized volumes. DSMC is a numerical method for approximating the Boltzmann equation. It was originally proposed for rarefied and transitional flows but has since been applied to near-continuum and continuum flows. DSMC can provide an accurate model for the entire boundary layer including the flow within the microstructure, where the size of the fibers may approach the mean-free-path of the flow. As the permeability of a porous material is a function of the distribution of its micropores, in this paper, the computational domain for DSMC simulations is obtained from three-dimensional (3D) images 
of the actual material microstructure acquired using X-ray microtomography (micro-CT). The technique allows to resolve the material into a 3D Cartesian mesh with a spatial resolution sufficient to discretize the fibrous structure.

A few examples can be found in the literature where rarefied flow simulations are applied to the determination of permeability of fibrous structures. White et al. [2] used the DSMC to investigate the permeabilities of two ablative materials, one cork-phenolic and one carbon-phenolic, in both their virgin and pyrolyzed states. They performed micro-CT scans of samples and imported the corresponding meshes for use in DSMC. They validated the method by comparing numerical results with experimental permeability measurements from a Berea sandstone material. The materials' permeabilities increased five to ten times following pyrolysis. They also found that the ablative materials were more permeable to argon than the pyrolysis gas, without investigating the presence of Klinkenberg effects in these materials. Stern et al. [3] computationally investigated the permeability of digitized fibrous materials generated from randomly oriented fibers at varying angles and porosities. Using DSMC the authors showed agreement with heritage data on refractory insulators. De Socio and 45 Marino [4] performed both experiments and numerical simulations to investigate the dynamics of gases in permeable media. The experimental effort consisted in applying a pressure differential across a stainless steel cylinder filled with glass spheres by manually controlling the mass flow rate, to reproduce rarefied conditions. The extracted pressure profile was compared with that obtained from two-dimensional DSMC simulations using a modified surface collision routine. De Socio and Marino [4] showed that a Knudsen-minimum type phenomenon was observed, meaning that there is a minimum in the measured permeability at a critical Knudsen number value, and higher permeability at Knudsen numbers lower and higher than the critical one. Kalarakis et al. [5] calculated the nitrogen gas permeability of a two-dimensional reconstructed porous media using two different techniques, the DSMC and a modified lattice-Boltzmann method, for Knudsen numbers in the 0.1-10 range. They reproduced the Klinkenberg equation very closely over the entire transition flow regime. Oshima et al. [6] 
performed DSMC simulations on nanoscale channels filled with randomly arranged solid spheres, in order to attain target values of the porosity. They showed that the particle diameter had a significant impact on the permeability even at fixed porosity. They also computed a mass flux proportional to the pressure gradient, which agrees with Darcy's law. Li and Sultan [7] studied the Klinkenberg slippage effect using a direct simulation Bhatnagar-Gross-Krook method and computed the gas permeability by pore-scale simulations at different pressures. They validated the Klinkenberg correlation model at low and high Knudsen numbers. In [8], Jaganathan et al. used 3D images of the microstructure of fibrous media obtained by digital volumetric imaging (DVI) to compute permeability using computational fluid dynamics (CFD). Results were 70 compared with analytical models of the normalized permeability as a function of the material volume fraction. The authors did not account for slip effects.

The present work is structured as follows. In section 2, we review the approximations used to model the mass flow through a fibrous material given a known pressure gradient in the continuum and Knudsen regimes. Two parameters, the effective permeability and the permeability slip parameter, are needed to fully close the approximations. In section 3, we summarize the method used to digitize porous structures and we describe the materials investigated. Section 4 introduces the numerical DSMC method used in this work. In section 5, we verify DSMC calculations of permeability with analytical solutions for 2D square arrays of cylinders at various Knudsen numbers. In section 6, we validate our approach by comparing results from simulations with values measured experimentally. We compare with data by Panerai et al. [9], who measured permeability data of FiberForm in Ar flow, at temperatures from 310 to 1320 K, using a flow tube experimental setup. FiberForm ${ }^{\circledR}$ (Fiber Materials, Inc., 85 Biddeford, Maine, USA) is the substrate for NASA's phenolic-impregnated carbon ablator (PICA), used in entry missions like Stardust and the Mars Science Laboratory. We show that the Darcy approximation and its Klinkenberg form are valid formulations for the materials that are studied. We also study different porous materials and show that the permeability is a strong function of 
the topology of the pores other than just the porosity of the material. Finally, section 7 summarizes the findings in the paper.

\section{Permeability of porous insulating materials}

The permeability of porous substrates for TPS materials, has been the subject of a limited amount of investigation. Marschall and Milos [10] performed permeability experiments on a range of rigid fibrous refractory insulators using a flow tube apparatus at NASA Ames Research Center. They forced a known mass flow rate of air through a cylindrical sample of material plugging the tube, and measured the pressure upstream and downstream of the material. They combined the ideal gas law, conservation of mass and the differential form of Darcy's law, to yield the relation

$$
\dot{m}=-\frac{A M}{\mu R T} K P \frac{d P}{d x},
$$

where $A$ is the cross-section area of the sample, $\dot{m}, \mu, M, T$ and $P$ are the mass flow rate, viscosity, molar mass, temperature and pressure of the gas, respectively, $K$ is the effective permeability and $R$ is the universal gas constant. Marschall and Milos [10] demonstrated that rarefied-flow effects for materials such as FiberForm are significant and that pressure dependence of the permeability is well expressed by the Klinkenberg form

$$
K=K_{0}(1+b / P)
$$

where $K_{0}$ is the material permeability in the limit of continuum flow, and $b$ is the permeability slip parameter that varies with temperature and gas viscosity. The quantity $b / P$ is a function of the local Knudsen number, and both $K_{0}$ and $b$ are dependent on the microstructure of the material [10]. $K_{0}$ and $b$ are both tensors, $\overline{\overline{K_{0}}}$ and $\overline{\bar{b}}$, that we assume diagonal since the samples were cut in directions aligned with their principal axes. Additionally, we assume that both components of the tensor in-plane are equal and we refer to them as $K_{0}^{\|}$and $b^{\|}$, respectively. Similarly, through the thickness of the material, the components 
of the permeability and slip parameter tensors are now written as $K_{0}^{\perp}$ and $b^{\perp}$. Marschall and Milos reduced the data and defined a permeability constant $F$, expressed as

$$
F=\frac{\mu \dot{m} R T L}{A M \Delta P}=K_{0}\left(P_{\text {avg }}+b\right)
$$

where $P_{a v g}$ and $\Delta P$ are the average pressure in the sample and the pressure difference across the sample, respectively. Since the present simulations are compared with data obtained using the same experimental method, the same convention is also adopted for our analysis. Additionally, they were able to derive, from an analogy for gas flow in a straight capillary tube with slip boundary condition, a scaling law for $b$ based on a measurement made at a reference gas composition and temperature as

$$
\frac{b(T, M, \mu)}{b^{*}}=\frac{\mu}{\mu^{*}} \sqrt{\frac{\pi}{2} \frac{R T}{M}} \sqrt{\frac{2}{\pi} \frac{M^{*}}{R T^{*}}}=\frac{\mu}{\mu^{*}} \sqrt{\frac{T}{T^{*}} \frac{M^{*}}{M}} .
$$
low-densiy carbon/phenolic ablators. The state-of-the-art material within this class is the NASA's phenolic-impregnated carbon ablator (PICA), which was successfully used during entry missions like Stardust (Earth reentry at $12 \mathrm{~km} / \mathrm{s}$ ) and the Mars Science Laboratory (MSL - Mars entry at $5.5 \mathrm{~km} / \mathrm{s}$ ). PICA ${ }_{140}$ is made through a resin-infusion process of FiberForm. A rendering of the $\left(M^{*}, \mu^{*}\right)$ at reference temperature $T^{*}$. Their data for FiberForm showed a significant amount of scatter between two groups of the four studied samples, likely due to large variations in the microstructure of FiberForm from batch to batch.

of the microstructure of the material. We will show using DSMC simulations that materials with the same porosity that have different microstructure distributions will have different permeability.

\section{Porous materials and microtomography imaging}

In this study we focus on fibrous materials that are used as substrates for (1) 
substrate is shown in Fig. 1a. Thanks to a NASA's technology transfer on infusion processing, SpaceX has also developed its own carbon/phenolic ablator (PICA-X). PICA-X, flight-proven during Dragon reentry from the ISS, uses Calcarb $\mathrm{CBCF}^{\circledR}[11]$ (Mersen, Courbevoie, France) (see Fig. 1d) as preform 145 material.

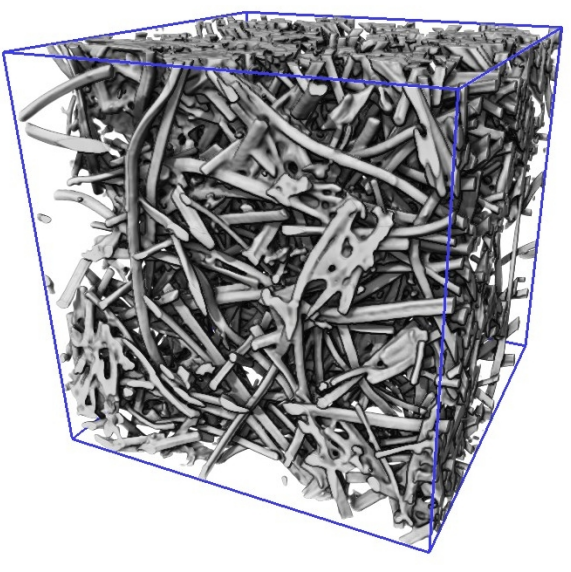

(a) Virgin FiberForm

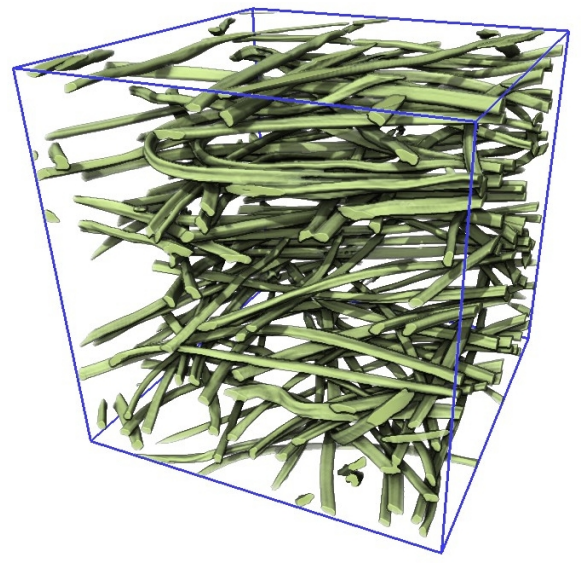

(c) Carbon Felt

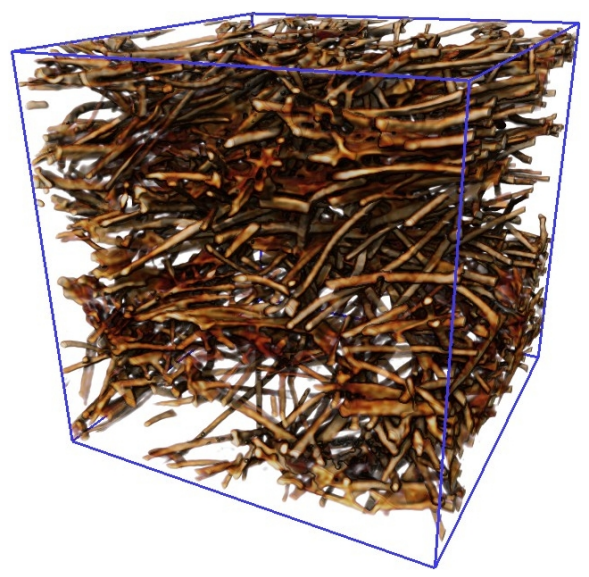

(b) Oxidized FiberForm

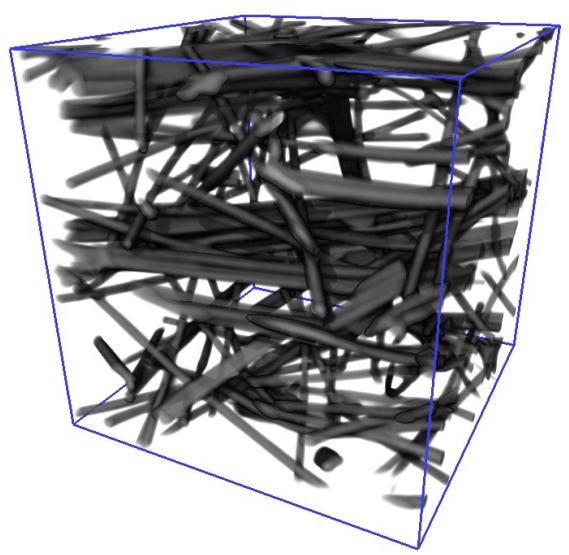

(d) Calcarb

Figure 1: Volume rendering of microtomography of some Thermal Protection Materials being investigated.

Both FiberForm and Calcarb are rigid materials, with average porosities 
higher than $85 \%$. Their micro-structure is characterized by fibers preferentially aligned at about $\pm 15^{\circ}$ with one of the planes (that perpendicular to the direction of compression during manufacturing). The arrangement of the fibers provides the material transverse isotropic properties, with the highest insulation capabilities (lowest thermal conductivity) in the "through-thickness" (TT) direction.

Examples of variations around the same material class are conformal and flexible carbon/phenolic composites $[12,13]$. These are manufactured according to a similar infusion processing as that used in heritage rigid ablators. However, unlike PICA, these new materials adopt flexible felts as the substrate (see Fig. 1c). Felt substrates suitable for conformal ablators are made of ceramic, polymers or carbon fibers. The use of a flexible preform has multiple advantages, chiefly an improved strain-to-failure that allows for manufacturing of larger structures, with more complex geometries. While felts have transverse isotropic properties as rigid substrates, the possibility of conforming the material around a certain shape allows to align the fiber with the surface, and therefore achieve the best insulation performance over the entire shape.

In this work we investigated a sample of virgin FiberForm, a sample of FiberForm that was oxidized by pure oxygen at $1200 \mathrm{~K}$ in NASA Ames flowtube reactor [14] (see Fig. 1b), and samples of Calcarb and Morgan carbon felt (Morgan Advanced Materials, Fostoria, Ohio, USA). This felt is one of the candidate precursors for the manufacturing of conformal PICA (C-PICA) [13]. The samples were cut from large billets of materials in the TT direction.

In order to produce a digital representation of the materials' microstructure, we acquired micro-CT images at the Advanced Light Source (ALS) at Lawrence Berkeley National Laboratory. We extracted samples by cutting material billets in the principal manufacturing directions. The specimens were place onto the tomography stage, aligning the sample with the axis of the stage. There are small unavoidable errors associated with cutting and installation operations, however we used our best practice to minimize them. Scans were performed at a X-ray energy of $14 \mathrm{keV}$, acquiring 1024 radiographs over a $180^{\circ}$ rotation for 
each scan. The projections were reconstructed as voxelized images, and then segmented using the same work-flow described in Ref. [15]. The tomography reconstruction process was based on a Fiji [16] plugin developed in-house at the ALS. The plugin was used to set input parameters for the Octopus reconstruction software [17] that applied the classical filtered back-projection algorithm by Kak and Slaney [18] for reconstruction and a removal algorithm for ring artifacts [19]. A 3D bilateral filter, developed for tomography images [20], was finally applied to the reconstructed data to improve edge-detection and reduce noise. The resulting filtered tomographies presented a bi-modal histogram of gray-level intensities. Segmentation was performed by choosing a single threshold value in correspondence of the local minimum in between the two modes of the histogram. The segmentation allowed to produce binary 3D images, where each voxels within the void phase of the microstructure is represented as black (gray level 0) and each material (carbon) voxel is represented as white (gray level 255). The quality of the segmentation was assessed by comparing tomography visualizations of each material with information available form scanning electron micrographs.

Micro-CT scans were performed by accurately placing the sample onto the imaging stage, in order to minimize the misalignment between the TT axis and the axis of rotation of the stage itself. Computational domains for DSMC simulations were prepared by extracting cubic volumes $(\approx 520 \mu \mathrm{m}$ edge $)$ of similar cell size.

200

Image renderings of the four samples are presented in Fig. 1. The figures were obtained by processing the micro-CT images in the Avizo 7.0 software (FEI Visualization Sciences Group, Burlington MA, USA). It can be qualitatively observed that the virgin FiberForm sample used here is significantly less porous than the virgin samples of felt and Calcarb. Due to the anisotropy of the materials, as for the thermal conductivity, the permeability will differ whether the gas flow in the TT direction of the material or in the normal ("in-plane", IP) direction. 

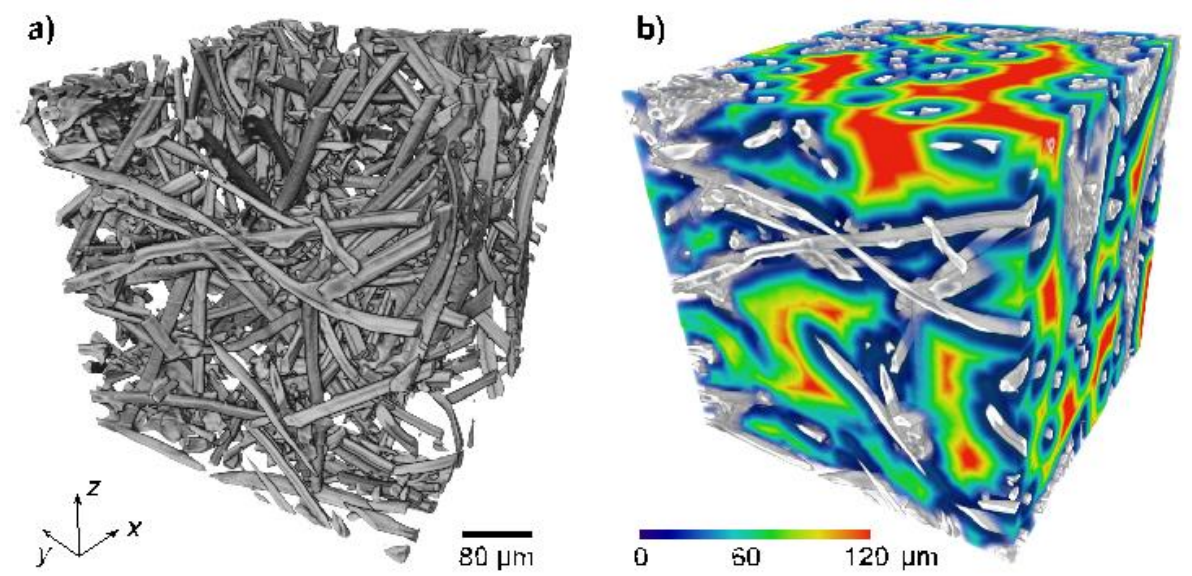

Figure 2: a) Microtomography rendering of FiberForm and b) correspondent distance field for the inter-fiber pores

\section{DSMC frame and computational domains}

It has been highlighted in a previous work [10] that rarefied-flow effects are significant in porous materials operating in high temperature, low pressure environments, and that using the Klinkenberg formulation for the permeability allows accounting for slip effects. For high temperature gases percolating through the microstructure of FiberForm-like materials, the local Knudsen number can be defined as a ratio of the gas mean free path, $\lambda$, to the mean pore diameter, $d_{p}$, assumed to be the characteristic length for the porous medium.

Figure 2a presents an example of the FiberForm microstructure rendered in Avizo together with a distance map (Fig. 2b) calculated for the voxels of the inter-fiber pores. The color field quantifies the distance of each voxel from the nearest object and allows to compute a characteristic mean pore diameter of $d_{p}$ ${ }_{220}=55 \mu \mathrm{m}$. As an example, the mean free path for oxygen in air can be estimate (in $\mu \mathrm{m}$ ) as [21]:

$$
\lambda=0.095 \frac{10^{5} \mathrm{~T}}{298 P}
$$

where $P$ is the average pressure. At flow temperatures between 1000 and $2000 \mathrm{~K}$ and pressures between 1 and $10 \mathrm{kPa}$, Knudsen numbers vary between 0.1 and 2, 


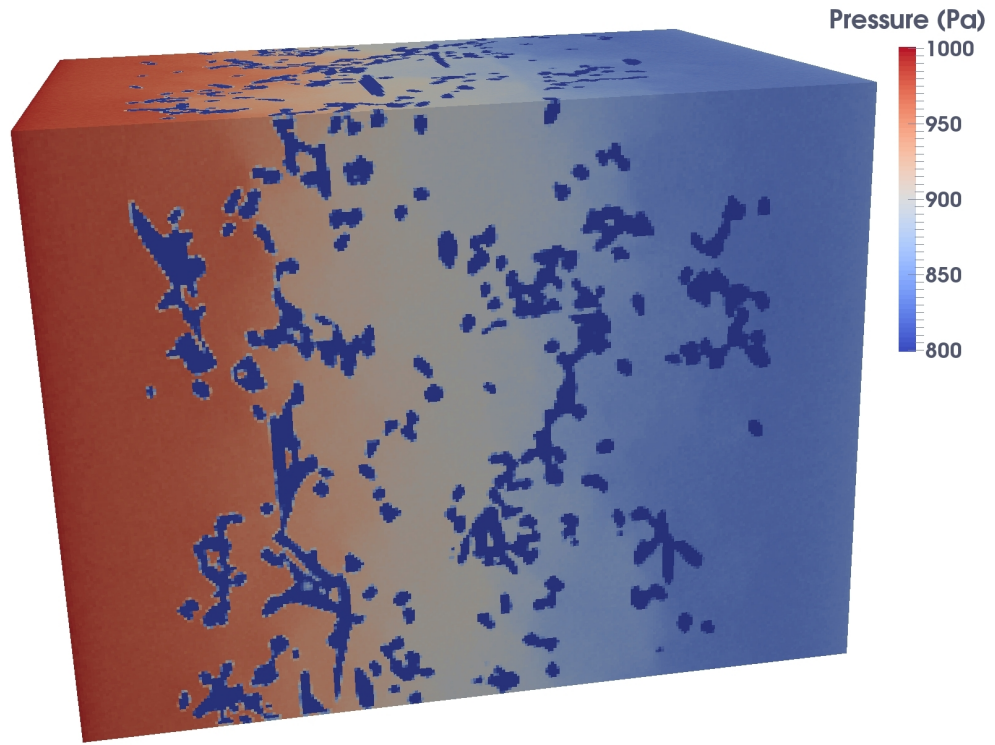

(a) Transverse

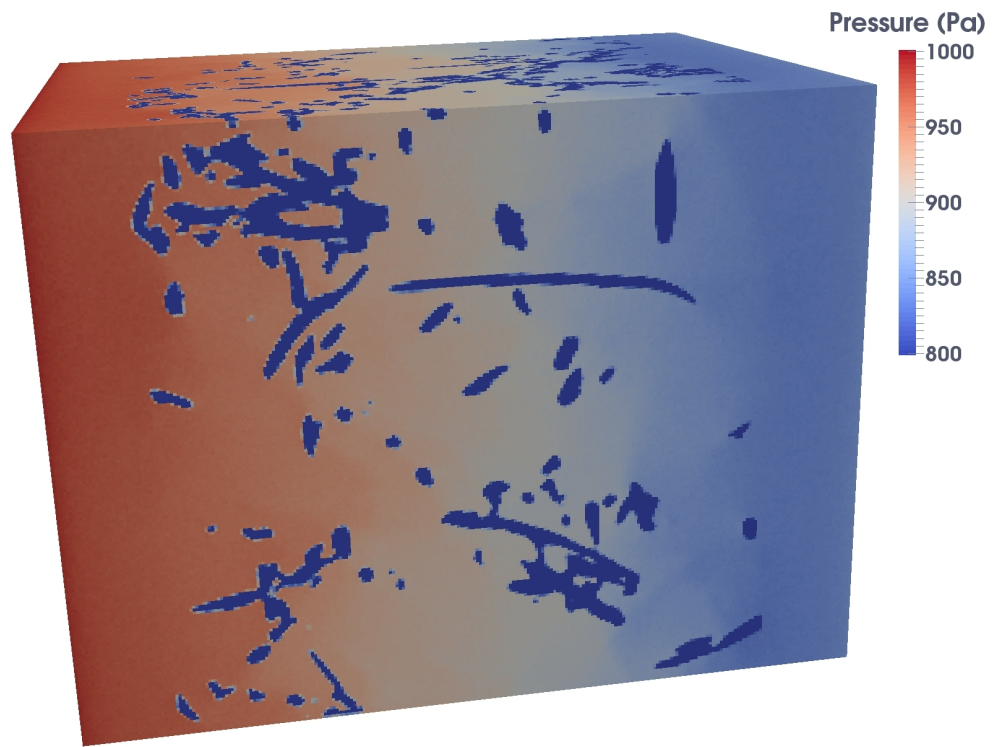

(b) In-Plane

Figure 3: Pressure for the flow of argon through FiberForm, for a temperature of $1319 \mathrm{~K}$ and an average pressure of $900 \mathrm{~Pa}$. Dark blue spots represent the location of the fibers. 
which are indication of a slip regime, and justify the use of DSMC simulations.

DSMC is a numerical Monte Carlo method to solve the time-dependent nonlinear Boltzmann equation. It is a probabilistic simulation of molecular processes based on the kinetic theory of dilute gases [1]. The general Boltzmann equation follows the following form

$$
\frac{\partial f}{\partial t}+\frac{\vec{p}}{m} \cdot \nabla f+\vec{F} \cdot \frac{\partial f}{\partial \vec{p}}=\left(\frac{\partial f}{\partial t}\right)_{\text {coll }},
$$

where $f$ is a probability density function, $\vec{p}$ is the momentum vector, $\vec{F}$ is the force field acting on the particles in the fluid and $m$ is the mass of the particle. The second term on the left-hand side represents the diffusion of the particles, the third term on the left-hand side represents the forces exerted on the particles by an external influence, and the term on the right-hand side is the collision term, accounting for the forces acting between particles in collisions. In the DSMC algorithm, the Boltzmann equation is decoupled into two parts: molecular advection and collisions. Particles are tracked in the domain based on their position in virtual cells. Each particle represents an $F_{N}$ number of real molecules. The cell length has to be carefully chosen such that it is a fraction of the mean free path. The time step is chosen such that it is a fraction of the mean collision frequency. At each time step, following their advection, a number of binary pairs of particles are selected for collisions in each grid cell. In the collision procedure, we follow the no-time-counter (NTC) procedure of Bird [1] for the selection of collision pairs. Quantities such as velocity, translational and internal energy of particles can be computed by sampling the flow field. Therefore, macroparameters such as temperature, pressure and number density can be computed in each grid cell. In order to reduce statistical scatter and ensure that collisions are performed accurately, it is generally recommended that a minimum of 10 to 20 particles per computational cell be used [22].

The Stochastic PArallel Rarefied-gas Time-accurate Analyzer (SPARTA) [23] DSMC code developed at Sandia National Laboratories was used in the current work. The grid was used to group particles by grid cell for purposes of performing collisions and chemistry, as well as efficiently find particle/surface collisions. 
A marching cubes algorithm [24] was implemented in SPARTA to approximate the isosurface of the micro-CT digitized geometry, given a grayscale threshold. The algorithm represents the surface of the fibers as a collection of triangles. These were used to locate surface collisions with particles during the DSMC simulation.

Subsonic boundary conditions were imposed to keep the pressure at the inlet and outlet planes of the domain, creating two buffer zones with constant quantities. 


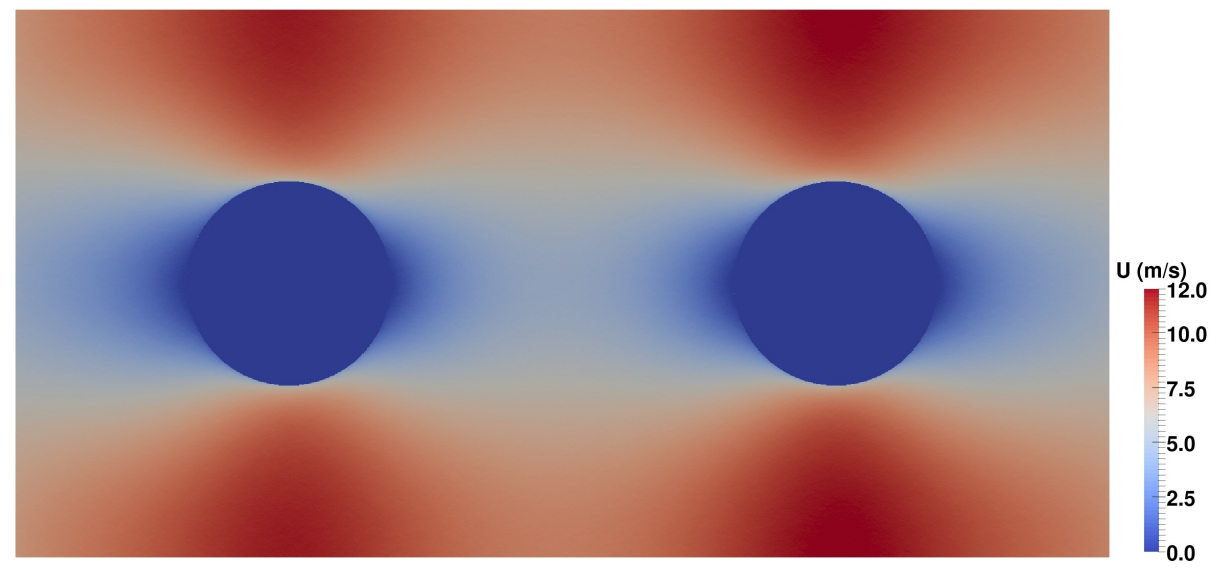

Figure 4: Contour plot of the $\mathrm{X}$ component of the velocity vector for the square array configuration, for a porosity of 0.89 and a Knudsen number of 0.01 .

\section{Permeability of square arrays}

In order to validate the method used to compute the permeability of porous media, we reproduced a classical problem of the flow through a square array of fibers. This problem has been investigated experimentally, numerically and theoretically by several authors $[26,27,28,3]$. Typically, most theoretical work attempt to relate the non-dimensional Darcy permeability (permeability divided by the square of the fiber radius) to the porosity of the structure. Shou et al. [26] derived an analytical equation to compute the non-dimensional permeability as a function of the porosity at different Knudsen numbers for the flow perpendicular to a $1 \mathrm{D}$ fibrous media as

$$
\frac{K}{r^{2}}=\frac{-0.5 \ln (1-\epsilon)+0.25-\epsilon-0.25(1-\epsilon)^{2}+2 \mathrm{Kn}\left(-0.5 \ln (1-\epsilon)-0.25+0.25(1-\epsilon)^{2}\right)}{4(1-\epsilon)(1+2 \mathrm{Kn})} .
$$

This equation was derived using a unit cell method from Stokes equation with a first-order slip boundary condition. Stern et al. [3] also computed the same problem using CFD and DSMC and compared it with an analytical expression. They found good agreement between the two numerical methods for Knudsen numbers up to 0.02 , and showed that the results started to diverge at a Knudsen number of 0.2 , which lies in the translational regime. 
Here, we set up a similar simulation as Stern's [3]: we model an infinite array considered first. Figure 3a shows the pressure field within our computational 


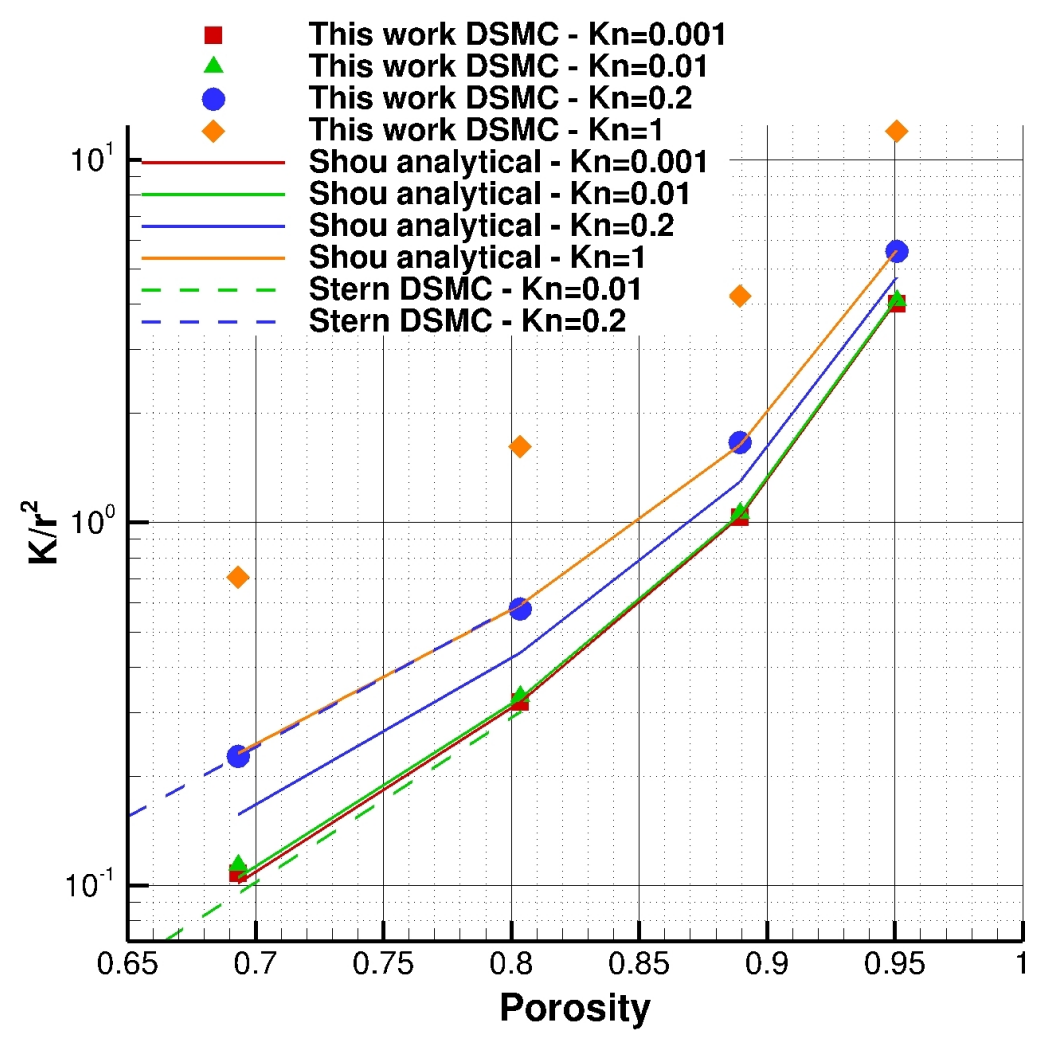

Figure 5: Non-dimensional permeability versus porosity for various Knudsen numbers, from DSMC, shows by symbols. DSMC results from Stern [3] and analytical results from Shou [26] are shown by dashed line and solid lines, respectively.

domain. The dark blue areas represent the location of carbon fibers, whereas the dark red to light blue gradient represents the pressure drop in the gas flow.

330 Figure 6 shows a comparison between the DSMC values for the permeability of an sample measured in the direction through the thickness, and the experimental data from Panerai et al. [9], at four temperatures. It can be seen that the agreement between computations and simulations is excellent, with the values of $K_{0}^{\perp}$ and $b^{\perp}$ showing less than a $2 \%$ difference at any temperature compared to experiments. Additionally, Table 1 shows a comparison between the values of the permeability parameters obtained from DSMC, and those normalized using 
the b-scaling law presented in Ref. [10]. It can be seen that the continuum flow permeability $K_{0}^{\perp}$ is not affected by $\mathrm{T}$ (less than $0.3 \%$ variation is computed between the high temperature simulation and the reference temperature $T^{*}=$ $310 \mathrm{~K})$. The $b^{\perp}$ parameter follows the scaling law exactly, with under $0.6 \%$ between the predicted value and the calculated one.

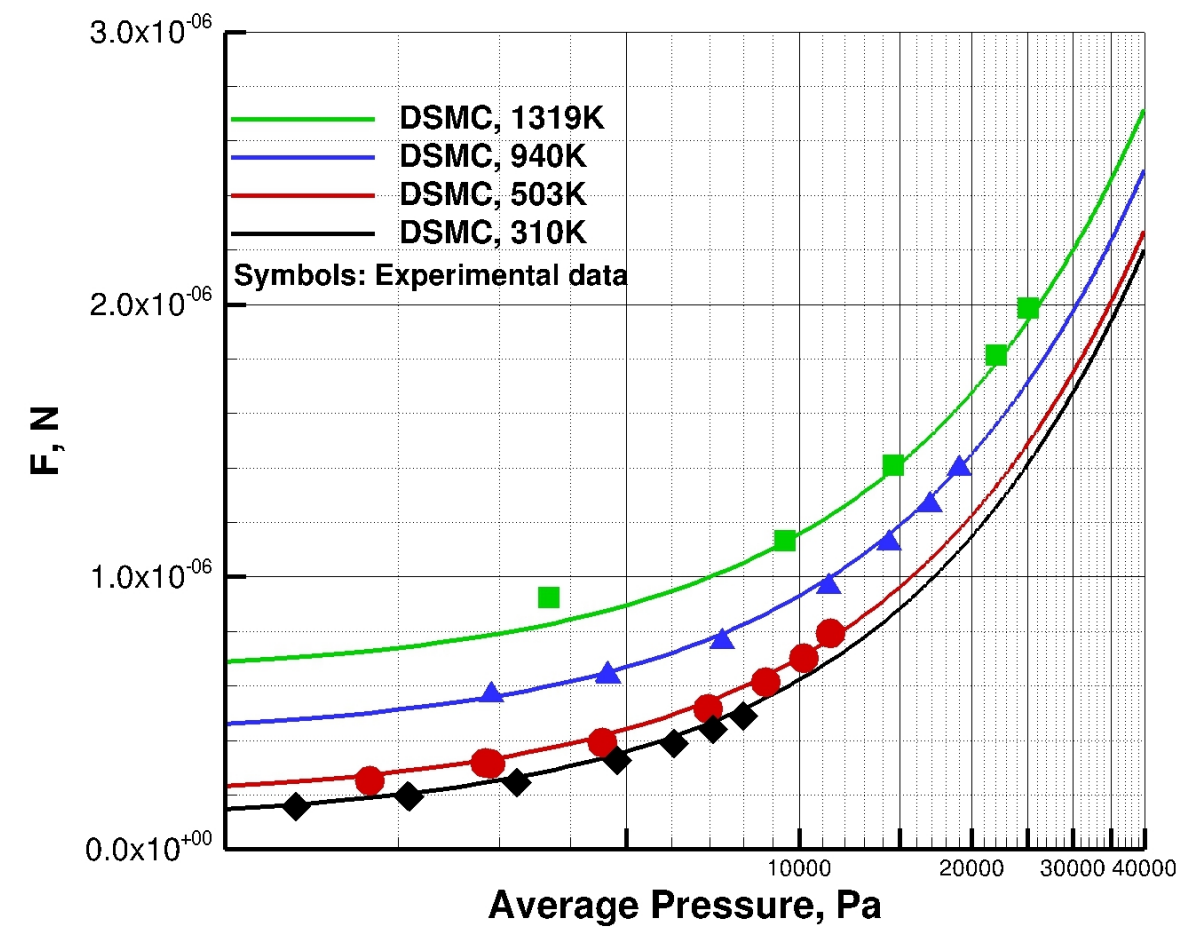

Figure 6: Permeability parameter $F$ for a transversely oriented virgin FiberForm specimen computed at four different temperatures, and comparison with experimental results.

Fig. 3b, Fig. 7 and Table 2 show similar data for the in-plane direction, also showing a good scaling with temperature. It is interesting to note that the continuum permeability value is nearly twice that of the through thickness direction in this case, emphasizing the othrotropy of FiberForm and impact it has on the flow of hot gases: gases flow slower in the direction parallel to 


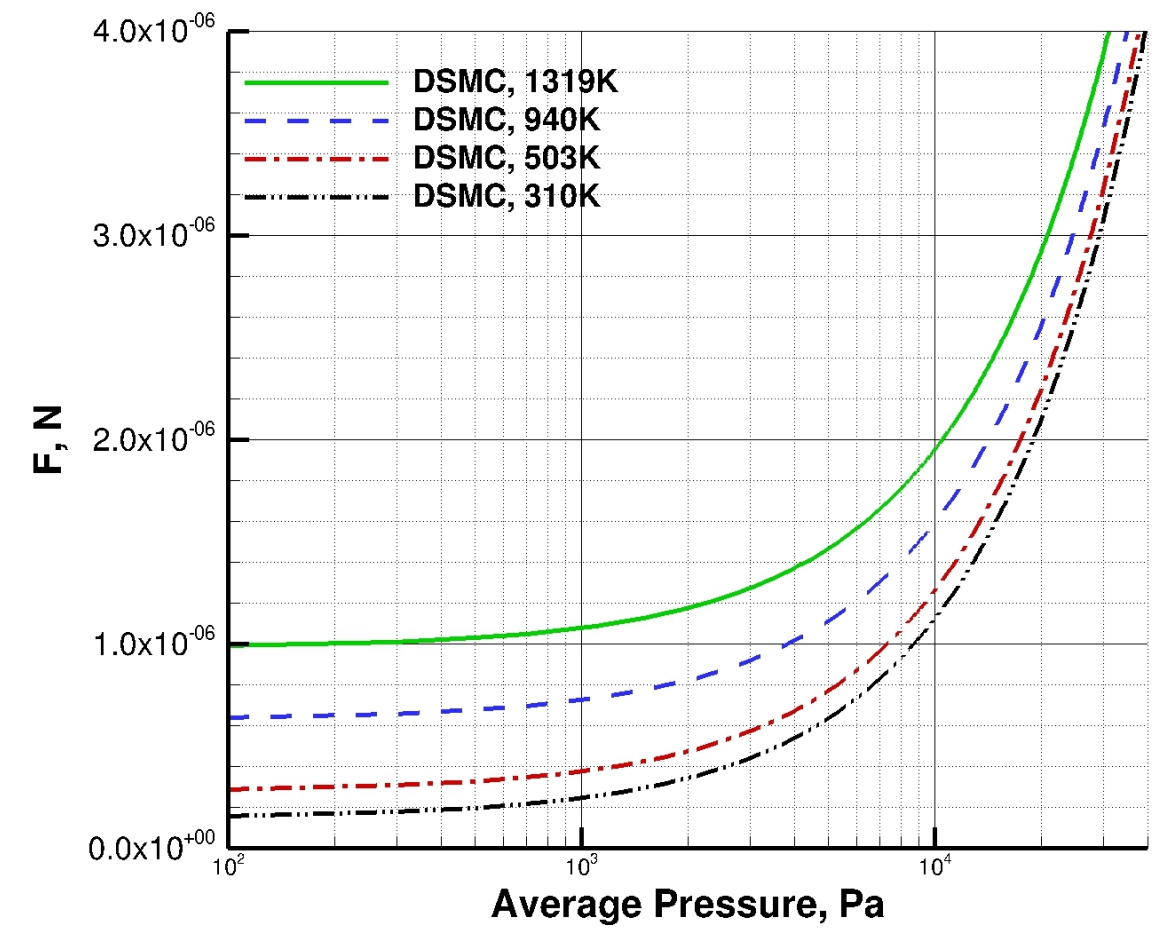

Figure 7: Permeability parameter $F$ for a planar oriented virgin FiberForm specimen computed at four different temperatures.

Table 1: Computational results for temperature dependent-simulations in the throughthickness direction.

\begin{tabular}{ccccc}
\hline \hline$T, \mathrm{~K}$ & $K_{0}^{\perp}, 10^{-12} \mathrm{~m}^{2}$ & $b^{\perp}, \mathrm{Pa}$ & $K_{0}^{\perp}(T) / K_{0}^{\perp}\left(T^{*}\right)$ & $\left(b^{\perp} / \mu \sqrt{T}\right) /\left(b^{* \perp} / \mu^{*} \sqrt{T^{*}}\right)$ \\
\hline 310 & 52.09 & 1831.0 & 1.000 & 1.000 \\
503 & 52.25 & 3432.4 & 1.003 & 0.994 \\
940 & 52.15 & 7817.5 & 1.001 & 0.998 \\
1319 & 52.08 & 12198.5 & 0.9997 & 0.9995 \\
\hline \hline
\end{tabular}

the pressing axis. Additionally, in Fig. 8 we show that, if $\mathrm{F}$ is normalized according to $F^{*} / K_{0} b^{*}$ and plotted as a function of $P_{\text {avg }} / b^{*}$, with $F^{*}$ computed 
Table 2: Computational results for temperature dependent-simulations in the planar direction.

\begin{tabular}{ccccc}
\hline \hline$T, \mathrm{~K}$ & $K_{0}^{\|}, 10^{-12} \mathrm{~m}^{2}$ & $b^{\|}, \mathrm{Pa}$ & $K_{0}^{\|}(T) / K_{0}^{\|}\left(T^{*}\right)$ & $\left(b^{\|} / \mu \sqrt{T}\right) /\left(b^{* \|} / \mu^{*} \sqrt{T^{*}}\right)$ \\
\hline 310 & 97.54 & 1517.5 & 1.000 & 1.000 \\
503 & 99.48 & 2783.4 & 1.010 & 0.986 \\
940 & 97.66 & 6439.9 & 1.001 & 0.992 \\
1319 & 97.63 & 10037.5 & 1.0009 & 0.992 \\
\hline \hline
\end{tabular}

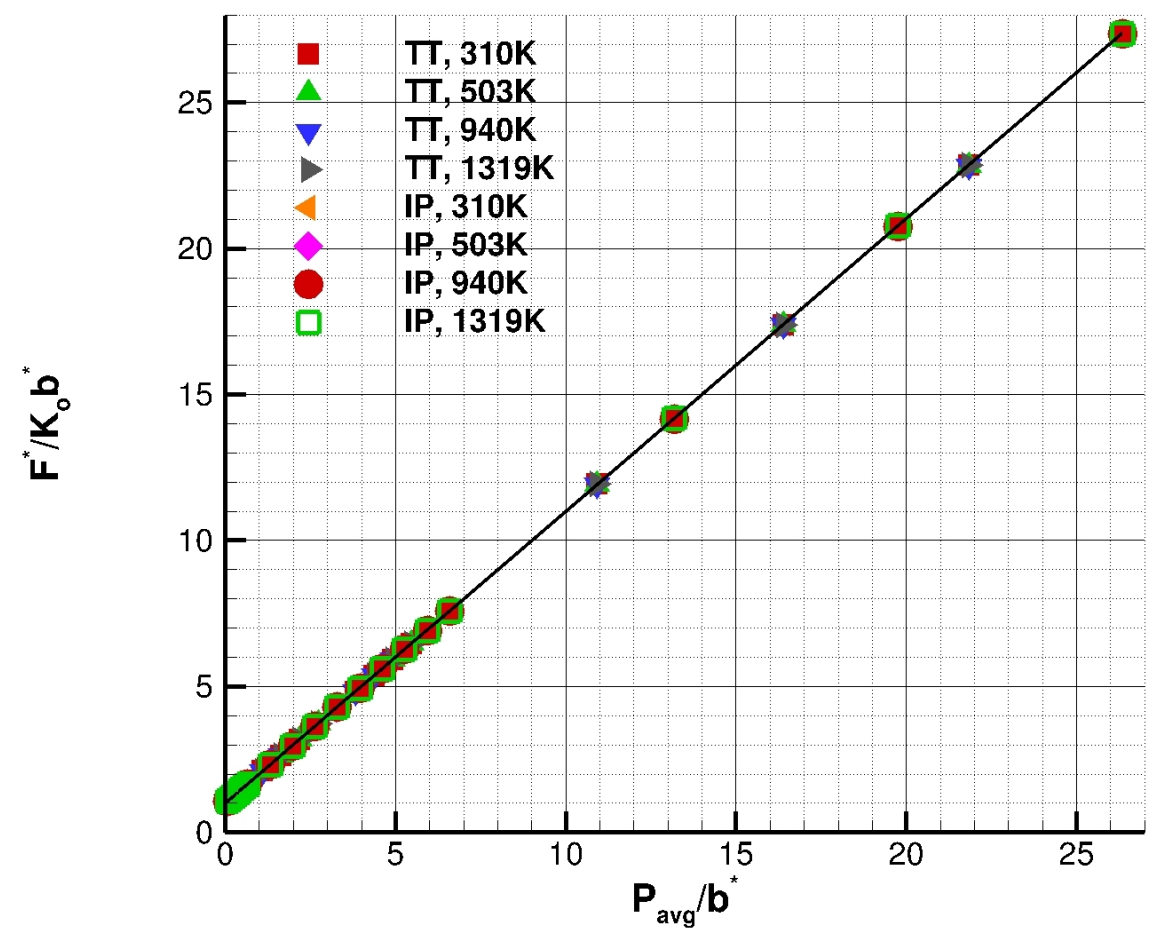

Figure 8: Normalized data from Figs. 6 and 7, show that all data collapse onto a single line.

by $F^{*}=K_{0}\left(P_{\text {avg }}+b^{*}\right)$ and $b^{*}$ the reference value for $b$ computed at $T^{*}=310 \mathrm{~K}$, all the curves in Figs. 6 and 7, collapse onto a single line.

The influence of the gas composition on the permeability was also inves- 
tigated. Similar simulations as the one conducted with a flow of argon were reproduced with flows of helium and air, at the reference temperature of $310 \mathrm{~K}$. It is interesting to note that FiberForm is more permeable to helium, which has smaller lighter molecules compared to argon or air. Table 3 shows a comparison of the viscosities, molar mass, and viscosity-temperature exponents (used in DSMC to model collisions with the VHS model), as well as the ratio of $K_{0}^{\perp}$ for helium and air with respect to argon, and the computed value of $b^{\perp}$ with the one extrapolated from the scaling law using argon as the reference gas. It can once again be seen that the behavior is completely predicted by the scaling law, and the three lines would perfectly superimpose if they were normalized in similar fashion to Fig 8. This makes it possible to study the influence of different atmospheres of different compositions and temperatures, without necessarily needing to conduct experiments or simulations with that atmosphere; the permeability value can simply be extrapolated from a measurement or calculation using a noble gas like argon.

Table 3: Computational results for gas dependent-simulations in the through-thickness direction. $d_{r e f}$ and $\omega$ are the reference diameter and viscosity temperature exponent in the VHS, respectively.

\begin{tabular}{cccccccc}
\hline \hline Gas & $\mu_{\text {ref }}$, Pa.s & $d_{\text {ref }}, \mathrm{m}$ & $\omega$ & $K_{0}^{\perp}, \mathrm{m}^{2}$ & $b^{\perp}, \mathrm{Pa}$ & $\frac{K_{0}^{\perp}(T, M, \mu)}{K_{0}^{\perp}\left(T^{*}, M^{*}, \mu^{*}\right)}$ & $\frac{\left(b^{\perp} / \mu \sqrt{T}\right)}{\left(b^{* \perp} / \mu^{*} \sqrt{T^{*}}\right)}$ \\
\hline Argon & $2.117 \times 10^{-5}$ & $4.17 \times 10^{-10}$ & 0.81 & $52.09 \times 10^{-12}$ & 1831.0 & 1.000 & 1.000 \\
Air & $1.719 \times 10^{-5}$ & $4.19 \times 10^{-10}$ & 0.77 & $51.51 \times 10^{-12}$ & 1752.7 & 0.989 & 1.014 \\
Helium & $1.865 \times 10^{-5}$ & $2.33 \times 10^{-10}$ & 0.66 & $51.32 \times 10^{-12}$ & 5074.1 & 0.985 & 1.008 \\
\hline \hline
\end{tabular}

In Fig. 9, we investigated the influence of porosity on permeability and the Klinkenberg parameters. While we have shown a good agreement with experiments, it is acknowledged that capturing the actual variability of the material at large scales with tomography volumes of the order a cubic millimeter is a challenging task. The local porosity of the material can significantly affect the computed permeability value. To address this aspect of the problem the porosity of the computational domain was adjusted by altering the cutoff for the 
marching cube algorithm. In varying the threshold value we ensured that the microstructure of the sample was similar, but fibers and pores sizes varied relatively continuously. The range of porosity that was investigated was 0.8 to 0.9 , which is a reasonable variability for FiberForm. The results for both $K_{0}$ and $b$ are plotted, and both datasets were fit with an exponential curve. Previous work on permeability of porous material proposed two sets of fits: one using a proportional to $\frac{\epsilon^{3}}{(1-\epsilon)^{2}}$ [29], and one proportional to an exponential curve [30]. Only the latter was able to properly fit our data for FiberForm. The following fit was obtained for $K_{0}: K_{0} \propto \exp (2.961 \epsilon)$. It is interesting to note that Ehrlich and Schwille [31] mention that the two Klinkenberg parameters are likely correlated, making it harder to analyze and fit them separately.

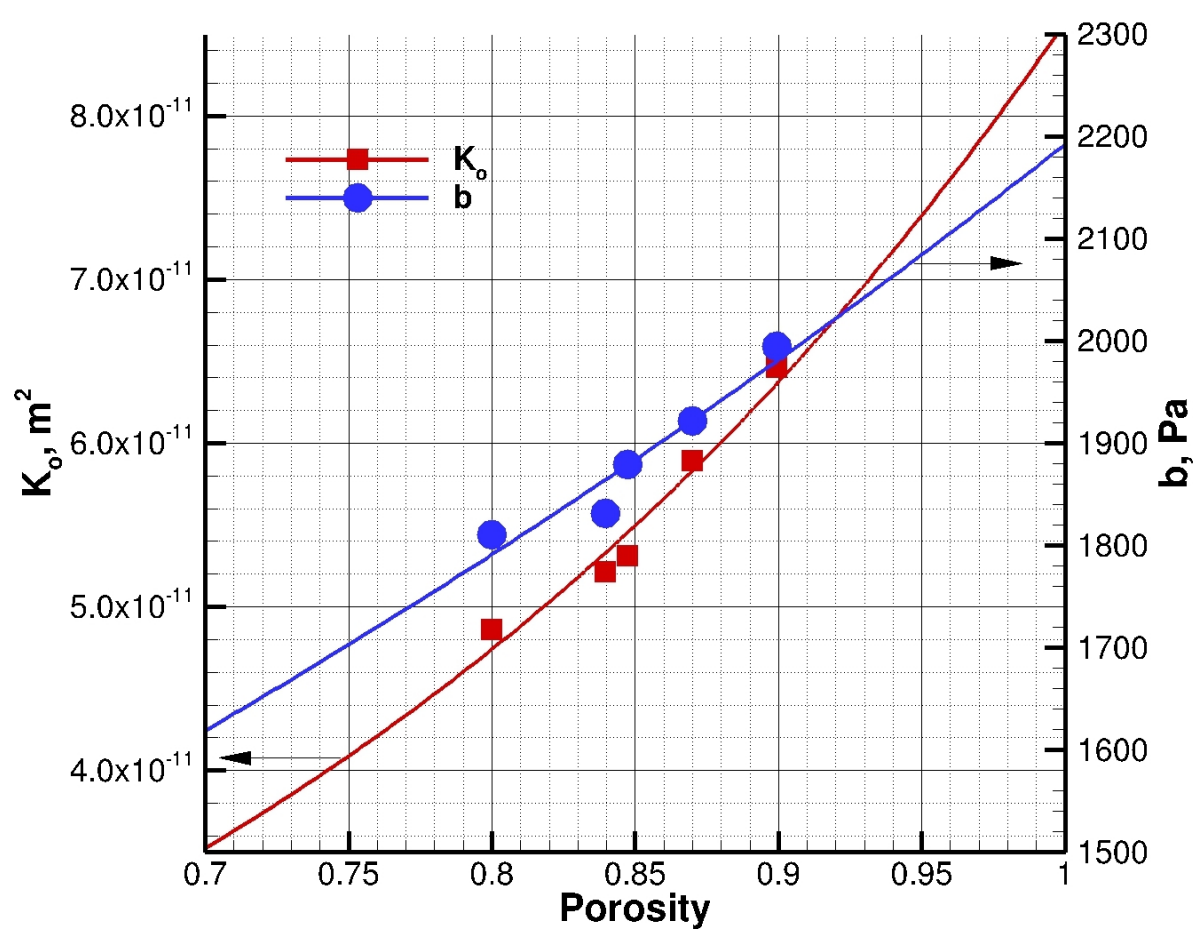

Figure 9: Permeability parameters $K_{0}$ and $b$ for a transversely oriented virgin FiberForm specimen for which the porosity was artificially varied by modifying the cutoff. 

made in Sec. 4 is confirmed by the porosity values presented in Table 4, along with the two permeability parameters computed for each material, $K_{0}^{\perp}$ and $b^{\perp}$, and the value of the permeability constant in the continuum. The latter was computed using the FlowDict module of the Geodict software [32], solving ary conditions are the same as those used in SPARTA. The values in the limit of the continuum are within $10 \%$ of the $K_{0}^{\perp}$ value obtained in DSMC simulations. The oxidation of the virgin FiberForm increased the material permeability by a fraction, and the values $K_{0}^{\perp}$ and $b^{\perp}$ for the oxidized sample of porosity 0.876 of FiberForm for which the porosity was artificially increased to 0.87 . On the other hand, the carbon felt and Calcarb both exhibit significantly higher permeabilities than the virgin and oxidized FiberForm. It should first be noted than both virgin materials have porosities larger than 0.90 , significantly above those ity, Calcarb exhibits a higher permeability than the carbon felt. That is due to the different microstructures of the two materials. Visually, Calcarb shows larger fibers and larger pores, while the felt is made out of thinner fibers and smaller pores. Additionally, both micro-CT and scanning electron micrograph trile (PAN) fibers within the material structure (see Fig. 11), and it has been shown computationally [33] that agglomeration increases the permeability at constant porosity. In support, Fig. 10 shows the volume fraction as a function of the pore size in the through-thickness direction, computed using a granulometry method in Geodict, that fits spheres of increasing sizes into the material pores. It can be seen that the average pore sizes for FiberForm, Morgan carbon felt and Calcarb are 50, 92 and $135 \mu \mathrm{m}$, respectively. The permeability of a porous material is therefore a function of the porosity as well as the pore size. 
Table 4: Computational results for porous thermal protection materials. Argon is used as the carrier gas and the temperature is fixed at $310 \mathrm{~K}$ in all cases. $K_{G e o}^{\perp}$ is the continuum permeability constant computed using Geodict.

\begin{tabular}{ccccc}
\hline \hline Material & Porosity & $K_{0}^{\perp}, 10^{-12} \mathrm{~m}^{2}$ & $b^{\perp}, \mathrm{Pa}$ & $K_{\text {Geo }}^{\perp}, 10^{-12} \mathrm{~m}^{2}$ \\
\hline Virgin FiberForm & 0.840 & 52.09 & 1831.0 & 46.71 \\
Oxidized FiberForm & 0.876 & 60.02 & 1908.5 & 55.31 \\
Carbon Felt & 0.939 & 195.43 & 1403.1 & 215.40 \\
Calcarb & 0.913 & 314.63 & 1001.5 & 315.50 \\
\hline \hline
\end{tabular}

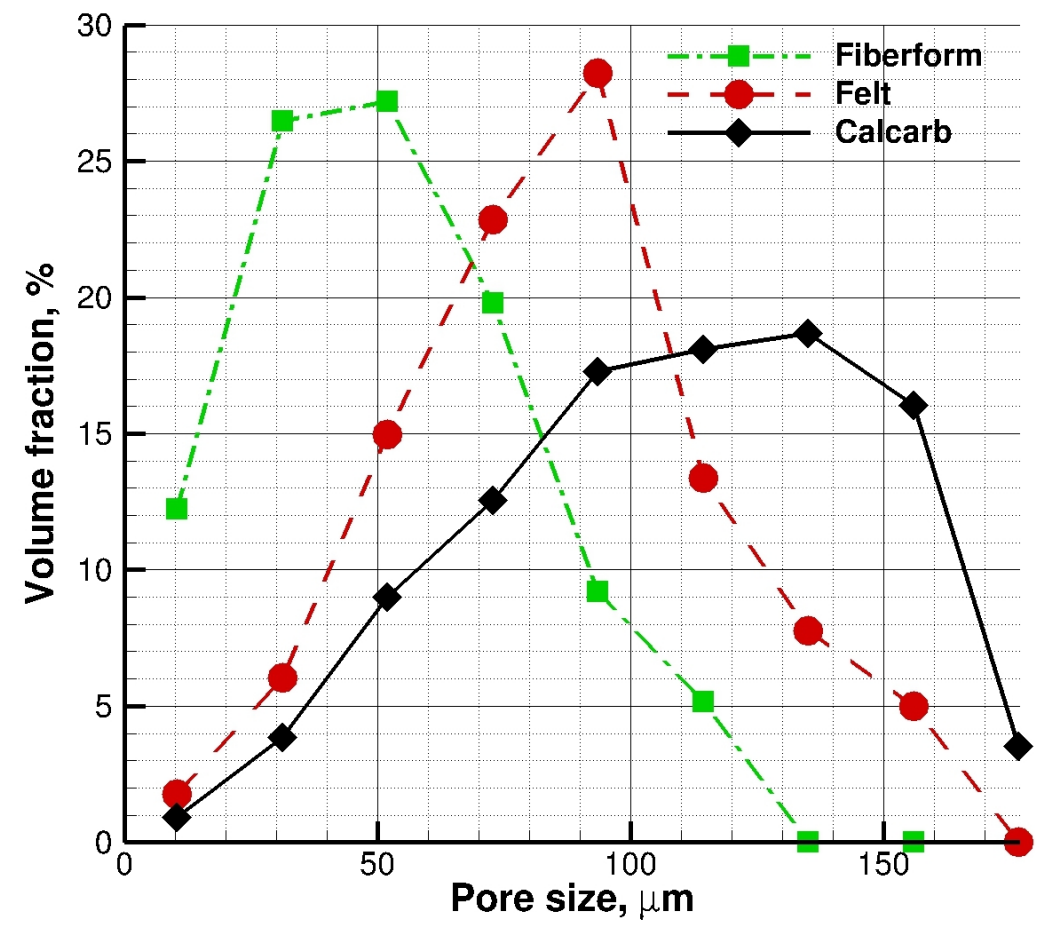

Figure 10: Volume fraction as a function of the pore size in the through-thickness direction for virgin FiberForm, Morgan carbon felt and Calcarb. 

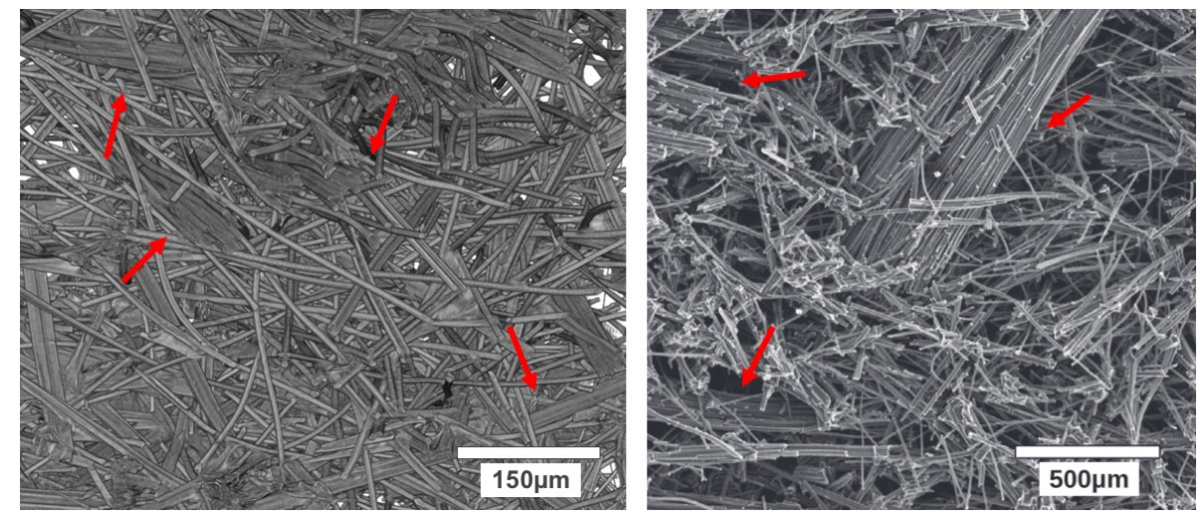

Figure 11: Micro-CT (left) and SEM (right) of Carlcarb. SEM picture reproduced from [34] with permission. Red arrows point at observed structures of agglomerated fibers.

\section{Conclusions}

A direct simulation Monte Carlo model was introduced to compute the permeability of porous thermal protection material in the slip regime, based on high-fidelity imaging of the material microstructure. The model is implemented in the SPARTA simulator and a marching cubes algorithm was added to the model to generate the virtual representation of the surface of the microstructure. Klinkenberg's form of the permeability constant was adopted following Marschall and Milos [10], such that the increased mass flow because of slip at the fibers' surface was accounted for.

Numerical computations were compared with experimental results for a sample of virgin FiberForm tested in the direction through the thickness and in the ${ }_{425}$ planar direction, at four different temperatures. Excellent agreement (under $2 \%$ difference) was reported, and Marschall's scaling law for temperature and gas composition was verified, meaning all the curves collapsed on a single one when normalized by the flow temperature. The continuum permeabilities for the transverse and in-plane orientation were $52.14 \pm 0.08$ and $98.08 \pm 0.94$ $\times 10^{-12} \mathrm{~m}^{2}$, respectively. Furthermore, the scaling was also verified when the gas composition was changed at fixed temperature. The influence of the porosity on permeability was investigated, and an exponential scaling law was fitted 
to the data in the case of FiberForm.

Finally, the permeability of the original virgin FiberForm sample was compared with that of a flow-tube oxidized sample of the same material, as well as virgin samples of Morgan carbon felt and Calcarb CBCF. An interesting observation was that the Calcarb exhibited significantly higher permeability than the carbon felt, even though its porosity was lower. Using a microscopic structure analysis, we concluded that this was due to the larger pore size in Calcarb.

\section{Acknowledgments}

The authors are indebted to Drs. M. Gallis and S. Plimpton (Sandia National Laboratories) for their assistance with SPARTA, and J. Ferguson (University of Kentucky) for fruitful discussions. Drs. A. A. McDowell and D. Y. Parkinson are gratefully acknowledged for their help in the LBNL/NASA Ames collaboration on micro-CT measurements. This work was performed under the Entry System Modeling Project (M. J. Wright project manager) of the NASA Game Changing Development (GCD) Program. This work was partially supported by NASA EAP Program administered by USRA. The Advanced Light Source is supported by the Director, Office of Science, Office of Basic Energy Sciences, of the U.S. Department of Energy under Contract No. DE-AC02-05CH11231.

\section{References}

[1] G. A. Bird, Molecular gas dynamics and the direct simulation of gas flows, Clarendon, 1994.

[2] C. White, T. J. Scanlon, R. E. Brown, Permeability of ablative materials under rarefied gas conditions, Journal of Spacecraft and Rockets (2015) $1-9$.

[3] E. C. Stern, I. Nompelis, T. E. Schwartzentruber, G. V. Candler, Microscale simulations of porous tps materials: Ablating microstructures and microtomography, AIAA paper (2015) 1450. 
[4] L. De Socio, L. Marino, Gas flow in a permeable medium, Journal of Fluid Mechanics 557 (2006) 119-133.

[5] A. Kalarakis, V. Michalis, E. Skouras, V. Burganos, Mesoscopic simulation of rarefied flow in narrow channels and porous media, Transport in porous media 94 (1) (2012) 385-398.

[6] T. Oshima, S. Yonemura, T. Tokumasu, A numerical study for transport phenomena of nanoscale gas flow in porous media, in: 28TH INTERNATIONAL SYMPOSIUM ON RAREFIED GAS DYNAMICS 2012, Vol. 1501, AIP Publishing, 2012, pp. 809-815.

${ }_{470}[7]$ J. Li, A. Sultan, Klinkenberg slippage effect in the permeability computations of shale gas by the pore-scale simulations, arXiv preprint arXiv:1505.01781.

[8] S. Jaganathan, H. V. Tafreshi, B. Pourdeyhimi, A realistic approach for modeling permeability of fibrous media: 3-d imaging coupled with $\{\mathrm{CFD}\}$ simulation, Chemical Engineering Science 63 (1) (2008) $244-252$. doi:http://dx.doi.org/10.1016/j.ces.2007.09.020.

URL http://www.sciencedirect.com/science/article/pii/ S0009250907007312

[9] F. Panerai, J. D. White, T. J. Cochell, O. M. Schroeder, N. N. Mansour, M. J. Wright, A. Martin, Experimental measurements of the permeability of fibrous carbon at high-temperature, International Journal of Heat and Mass Transfer 101 (2016) 267-273.

[10] J. Marschall, F. S. Milos, Gas permeability of rigid fibrous refractory insulations, Journal of thermophysics and heat transfer 12 (4) (1998) 528-535.

485 [11] Mersen (Brochure). CALCARB rigid carbon thermal insulation, http://www.mersen.com/uploads/tx_mersen/ 3-CALCARB-rigid-carbon-thermal-insulation-mersen_12.pdf (January 2015). 
[12] F. S. Milos, M. J. Gasch, D. K. Prabhu, Conformal phenolic impregnated carbon ablator arcjet testing, ablation, and thermal response, Journal of Spacecraft and Rockets 52 (3) (2015) 804-812. doi:10.2514/1.A33216.

URL http://dx.doi.org/10.2514/1.A33216

[13] M. Gasch, M. Stackpoole, S. White, T. Boghozian, Development of Advanced Conformal Ablative TPS Fabricated from Rayon- and Pan-Based Carbon Felts, American Institute of Aeronautics and Astronautics, 2016. doi:doi:10.2514/6.2016-1414.

URL http://dx.doi.org/10.2514/6.2016-1414

[14] F. Panerai, A. Martin, N. N. Mansour, S. A. Sepka, J. Lachaud, Flow-tube oxidation experiments on the carbon preform of a phenolic-impregnated carbon ablator, Journal of Thermophysics and Heat Transfer 28 (2) (2014) 181-190.

[15] J. C. Ferguson, F. Panerai, J. Lachaud, A. Martin, S. C. Bailey, N. N. Mansour, Modeling the oxidation of low-density carbon fiber material based on micro-tomography, Carbon 96 (2016) 57-65.

[16] J. Schindelin, I. Arganda-Carreras, E. Frise, V. Kaynig, M. Longair, T. Pietzsch, S. Preibisch, C. Rueden, S. Saalfeld, B. Schmid, et al., Fiji: an open-source platform for biological-image analysis, Nature methods 9 (7) (2012) 676-682.

[17] M. Dierick, B. Masschaele, L. V. Hoorebeke, Octopus, a fast and userfriendly tomographic reconstruction package developed in labview, Measurement Science and Technology 15 (7) (2004) 1366.

[18] A. Kak, M. Slaney, Principles of Computerized Tomographic Imaging, Society for Industrial and Applied Mathematics, 2001. doi:10.1137/1. 9780898719277.

515

[19] D. Prell, Y. Kyriakou, W. A. Kalender, Comparison of ring artifact correction methods for flat-detector ct, Physics in Medicine and Biology 54 (12) 
(2009) 3881.

URL http://stacks.iop.org/0031-9155/54/i=12/a=018

[20] D. Ushizima, T. Perciano, H. Krishnan, B. Loring, H. Bale, D. Parkinson, J. Sethian, Structure recognition from high resolution images of ceramic composites, in: Big Data (Big Data), 2014 IEEE International Conference on, 2014, pp. 683-691. doi:10.1109/BigData.2014.7004292.

[21] R. Reid, J. Prausnitz, B. Poling, The properties of gases and liquids (4th cd.) mcgraw-hill, New York.

[22] Z.-X. Sun, Z. Tang, Y.-L. He, W.-Q. Tao, Proper cell dimension and number of particles per cell for dsmc, Computers \& Fluids 50 (1) (2011) 1-9.

[23] M. A. Gallis, J. R. Torczynski, S. J. Plimpton, D. J. Rader, T. Koehler, Direct simulation monte carlo: The quest for speed, Proceedings of the 29th Rarefied Gas Dynamics (RGD) Symposium.

[24] W. E. Lorensen, H. E. Cline, Marching cubes: A high resolution 3d surface construction algorithm, SIGGRAPH Comput. Graph. 21 (4) (1987) 163169. doi:10.1145/37402.37422.

URL http://doi.acm.org/10.1145/37402.37422

[25] J. Ahrens, B. Geveci, C. Law, 36 paraview: An end-user tool for large-data visualization, The Visualization Handbook (2005) 717.

[26] D. Shou, J. Fan, F. Ding, Hydraulic permeability of fibrous porous media, International Journal of Heat and Mass Transfer 54 (17) (2011) 4009-4018.

[27] Z. Chai, J. Lu, B. Shi, Z. Guo, Gas slippage effect on the permeability of circular cylinders in a square array, International Journal of Heat and Mass Transfer 54 (13) (2011) 3009-3014.

[28] D. Shou, Gas and vapor transport through nano-and micro-fibrous materials, Ph.D. thesis, The Hong Kong Polytechnic University (2013). 
[29] R. B. Bird, Transport phenomena, Applied Mechanics Reviews 55 (1) (2002) R1-R4.

[30] R. Gauvin, A. Kerachni, B. Fisa, Variation of mat surface density and its effect on permeability evaluation for rtm modelling, Journal of Reinforced Plastics and Composites 13 (4) (1994) 371-383.

[31] D. Ehrlich, J. A. Schwille, Permeability of space vehicle fibrous thermal protection blankets in viscous and slip flow regimes, Journal of Aerospace Engineering 28 (3).

[32] A. Wiegmann, Computation of the permeability of porous materials from their microstructure by FFF-Stokes, Fraunhofer-Institut für Techno-und Wirtschaftsmathematik, Fraunhofer (ITWM), 2007.

[33] G. Bechtold, L. Ye, Influence of fibre distribution on the transverse flow permeability in fibre bundles, Composites Science and Technology 63 (14) (2003) 2069 - 2079, polymer Composites: Design, Materials, Manufacturing, Dedicated to Professor M. Neitzel. doi:http://dx.doi .org/10.1016/S0266-3538(03)00112-X.

URL http://www.sciencedirect.com/science/article/pii/ S026635380300112X

[34] B. Helber, O. Chazot, A. Hubin, T. E. Magin, Microstructure and gas-surface interaction studies of a low-density carbon-bonded carbon fiber composite in atmospheric entry plasmas, Composites Part A: Applied Science and Manufacturing 72 (2015) 96 - 107. doi:http://dx.doi.org/10.1016/j.compositesa.2015.02.004. URL http://www.sciencedirect.com/science/article/pii/ S1359835X15000469 